\title{
An increase in Accident and Emergency presentations for Adverse Events Following Immunisation after introduction of the group B meningococcal vaccine : an observational study.
}

\author{
Viveka Nainani1,2, Ushma Galal' ${ }^{3}$, Jim Buttery ${ }^{2,4,5}$, Matthew D. Snape ${ }^{1,6}$
}

10xford Vaccine Group, University of Oxford, Department of Paediatrics, Oxford, United Kingdom

2Department of Paediatrics, Monash University, Infection and Immunity, Monash Children's Hospital, Clayton, Victoria, Australia

${ }^{3}$ Nuffield Department of Primary Care Health Sciences, Clinical Trials Unit, University of Oxford, Oxford, United Kingdom

${ }^{4}$ SAEFVIC; Murdoch Childrens Research Institute; Melbourne, Victoria, Australia; ${ }^{5}$ Monash Immunisation; Monash Health; Clayton, Victoria, Australia

${ }^{6}$ NIHR Oxford Biomedical Research Centre, Oxford University Hospitals NHS trust, Oxford

Correspondence to: V Nainani

vknai2@student.monash.edu

Current word counts:

Abstract 257

Text 2553 


\section{Objectives}

To determine whether the introduction of the capsular group B meningococcal vaccine (4CMenB) in the United Kingdom has increased presentations of infants to Emergency Departments with Adverse Events Following Immunisation (AEFI)

\section{Participants, design and setting.}

A retrospective review of hospital records of 1-6 month olds presenting to Oxford University Hospitals NHS Trust's Emergency Departments from September 2013 to August 2016 with discharge diagnoses of vaccine reactions or non-specific conditions. Immunisation history was checked by reference to centralised immunisation records.

\section{Main outcome measures}

Presentation classifications were: 'probable vaccine reaction' (i.e. symptoms within 48 hours of immunisation; no alternative cause found); 'possible vaccine reaction' (symptoms within 48 hours of immunisation with a possible alternative cause) or 'not related' (clear alternative diagnosis or not immunised within previous 48 hours).

\section{Results}

Prior to 4CMenB introduction (2013-15), an annual average of 12 infants presented with probable or possible AEFIs, increasing to 38 infants in the year following 4CMenB introduction (2015/2016). Rates of AEFIs per 1000 immunisation episodes increased post4CMenB introduction from 1.03 to $3.4(\mathrm{p}<0.001)$ at 2 months and from 0.14 to 1.13 (p= 0.005) at 4 months. At 3 months, when $4 \mathrm{CMenB}$ is not given, no increase was seen $(p=0.380) .4 C M e n B$ introduction was also associated with increased AEFI-related hospital admissions, invasive investigations and intravenous antibiotic use. 


\section{Conclusions}

Further research is required to develop management guidelines for infants presenting with AEFIs following 4CMenB immunisation, as the increase in emergency department attendances, investigations and antibiotic use for AEFIs may influence the cost effectiveness of the 4CMenB immunisation campaign. 


\section{Introduction}

The capsular group B meningococcal vaccine $(4 \mathrm{CMenB})$ was introduced into the routine UK immunisation schedule at 2, 4 and 12 months of age in September 2015, with a catch up campaign for all infants born after $1^{\text {st }}$ May 2015. Immunisation coverage of over $90 \%$ was achieved, and the vaccine has demonstrated an effectiveness of $83 \%$ against capsular group B meningococcal disease. (1)

The vaccine is known to be relatively reactogenic, with $61 \%$ of infants developing a fever above $38^{\circ} \mathrm{C}$ when receiving a dose of $4 \mathrm{CMenB}$ administered concomitantly with routine vaccinations. (2) While not harmful in itself, this fever is associated with temporary distress in the child and can be concerning to parents, potentially resulting in them seeking medical assessment in emergency departments. Clinicians assessing these infants may then initiate invasive investigations or intravenous antibiotics given the low threshold for these interventions in febrile infants under 3 months of age.(3)

In light of this, and given evidence that rates of post-4CMenB fever are reduced by prophylactic paracetamol peri-immunisation, Public Health England have recommended the routine use of paracetamol at the time of immunisation and in the subsequent 24 hours. (4)

Nevertheless, the potential remains for the introduction of $4 \mathrm{CMenB}$ to lead to increased numbers of infants presenting to emergency departments with adverse events following immunisation (AEFIs), and we therefore evaluated these presentations and their management in the pre- and post-4CMenB era. 


\section{Materials and Methods}

This retrospective study was conducted as a service evaluation at Oxford University Hospitals NHS trust, which provides emergency paediatric care for all of Oxfordshire through the Accident and Emergency (A\&E) departments at the John Radcliffe hospital (a tertiary hospital) and at the Horton hospital (a district general hospital). The discharge summaries of all infants aged 1 to 6 months presenting to these A\&E departments between $1^{\text {st }}$ September 2013 and $31^{\text {st }}$ August 2016 were reviewed to detect potential AEFI's; a period two years before, and 1 year after, the introduction of the 4CMenB vaccine (Bexsero, GSK, Italy) on $1^{\text {st }}$ September 2015. This vaccine was introduced in a 2, 4 and 12 month schedule for babies born on or after $1^{\text {st }}$ July 2015, accompanied by a limited 'catch up campaign' for children presenting for their 3 or 4 month immunisations who were born after $1^{\text {st }}$ May 2015. Other routine immunisations administered in the UK at 2, 3 and 4 months of age are detailed in Supplementary Table 1. (5)

Discharge diagnoses of all 1-6 month old infants presenting to A and E were analysed to detect AEFIs, either through a discharge diagnosis of post-immunisation reactions, or with nonspecific presentations or diagnoses (e.g.: fever, irritability, rash, seizures, floppiness or being acutely unwell). If this assessment was supported by review of the electronic medical notes then the immunisation histories of these children were determined using the Oxfordshire Child Health Computer database to identify administration of any vaccines within the 48 hours prior to presentation. Children with a clear alternative diagnosis were not assessed further.

Children identified as having potential AEFIs in this manner were classified as having presentations that were: 'probably related to immunisation' (symptom onset within 48 hours of immunisation, no alternative focus found); 'possibly related to immunisation' (symptom 
onset was within 48 hours of immunisation, possible alternative focus found); or 'not related to immunisation' (symptoms were not within 48 hours of immunisation or a definite alternative focus was found). Classification of a 'serious' AEFI was conducted according to WHO criteria, and included inpatient hospitalisation (6).

The electronic records of all infants classified as having a probable or possible reaction were independently reviewed by a second investigator and any discrepancies (including classifying events as probable or possible) resolved by re-evaluating clinical notes, pathology results and re-checking immunisation histories from the Primary Care Child Health database.

Further clinical details of children classified as having a 'probable' or 'possible' vaccine reaction were obtained, including the performance of invasive investigations, admission to the ward or Clinical Decision Unit (CDU, a 24 hour observation facility) and use of intravenous antibiotics. Numbers of infants with 'probable' and 'possible' vaccine reactions were combined for subsequent analyses to ensure all AEFI's were included in the analyses.

The numbers of 1 to 6 month olds presenting with urinary tract infections (UTIs, identified by review of discharge summaries and confirmed by a positive urine microscopy or culture), were also determined and used as a condition that was unrelated to immunisation and could control for potential confounders such as changes in population numbers, referral patterns or health seeking behaviour.

\section{Patient involvement}

As this was a retrospective observational study, patients were not involved in the design or 
outcome of the study.

\section{Statistical analysis}

Infants presenting to A\&E before $1^{\text {st }}$ September 2015 (the date of $4 \mathrm{CMenB}$ introduction) were classified as belonging to the 'pre-4CMenB' era, while those from this date onwards were 'post4CMenB.'

The numbers of probable or possible AEFIs at the 2, 3 and 4 month immunisation episodes were divided by the number of immunisations given across Oxfordshire at these ages (obtained from the Oxfordshire Child Health computer database) to determine the rates of AEFIs per 1000 doses of the vaccine. In the pre-4CMenB era, the denominator used was the number of DTaP-IPV-Hib vaccines administered at 2, 3 and 4 months; in the post-4CMenB era, it was the number of doses of 4CMenB (2 months), DTaP-IPV-Hib (3 months) and 4CMenB (4 months). Confirmation that an AEFI occurring at 2, 3 and 4 months of age represented an association with the $1^{\text {st }}, 2^{\text {nd }}$ and $3^{\text {rd }}$ scheduled immunisation was made by reference to the child health computer database.

For each of the 2, 3 and 4 month immunisation episodes, as well as the combined 2 and 4 month episodes, the Chi-Square test was used to assess the association between 4CMenB era and presentation with possible or probable AEFI. Results are presented as odds ratios and 95\% confidence intervals with corresponding $\mathrm{p}$-values.

The rates of the clinical interventions (ward and CDU admissions, blood tests, lumbar punctures and intravenous antibiotics) were calculated per 1000 doses of vaccine for the 3 month and combined 2 and 4 month immunisation episodes. For each intervention, the 
association with $4 \mathrm{CMenB}$ era was tested using the Chi-square test and results are reported as odds ratios.

Children receiving $4 \mathrm{CMenB}$ at 3 months of age in the 'catch-up' campaign were analysed separately and excluded from the analysis of AEFIs across the 1 to 6 month age range to better represent likely AEFIs seen in an ongoing 'routine' immunisation schedule. In instances where expected numbers were too small for the Chi-square test to be valid, Fisher's Exact test was used. For all analyses, a p-value of $<0.05$ was considered significant. All data was analysed using the Statistical Package for the Social Sciences (SPSS) 22.

\section{Results}

\section{Presentations to $A \& E$}

Over a 3 year period, 5209 infants aged $1-6$ months presented to the John Radcliffe $(\mathrm{n}=$ 4626) and Horton hospitals $(n=583)$ with potential AEFIs (Supplementary Figure 1). A total of 62 probable or possible AEFIs were identified (Figure 1), all of which had their immunisation histories confirmed using the Primary Care Child Health database. The mean annual incidence of AEFI presentations prior to $4 \mathrm{CMenB}$ introduction was 12 per year compared with 38 in the year post-4CMenB introduction (24 out of 3342 presentations pre-4CMenB, 38/1867 presentations post- $4 \mathrm{CMenB}, \mathrm{p}<0.0001)$. In contrast, the numbers of presentations with UTIs in this age group remained stable (26.5 per year in the pre-4CMenB era and 24 in the year post4CMenB. In contrast, there was no statistically significant difference in the number of UTIs between the pre-4CMenB and post- $4 \mathrm{CMenB}$ eras $(53 / 3342$ and $28 / 1867$, respectively, $\mathrm{p}=$ $0.81)$ 
The rates of $\mathrm{A}$ and $\mathrm{E}$ presentations for AEFIs (possible and probable combined) per 1000 vaccines administered at 2, 3 or 4 months in Oxfordshire are shown in Table 1 and Supplementary Figure 2. These increased significantly in the post-4CMenB era following the immunisation episodes at 2 months $(1.03 / 1000$ to $3.4 / 1000)$ and 4 months $(0.14 / 1000$ to 1.13/1000). No similar increase was observed for the 3 month immunisation episodes, when no $4 \mathrm{CMenB}$ vaccine is administered in the routine schedule. Of note, when 3 month olds received $4 \mathrm{CMenB}$ together with routine vaccines in the 'catch-up' campaign, $6.76 \mathrm{AEFIs}$ were seen per 1000 doses; almost double the rate observed in 2 month olds in the post-MenB era. Overall there were 2.36 presentations for AEFIs per 1000 doses of 4CMenB administered during routine immunisation at 2 and 4 month immunisation episodes, compared with 0.59 per 1000 doses at these times in the pre- $4 \mathrm{CMenB}$ era $(\mathrm{OR}(95 \% \mathrm{CI})=4.0(2.2,7.2)$, p-value $<0.001)$.

\section{Clinical interventions following routine $4 \mathrm{CMenB}$ immunisation at 2 and 4 months of age}

Following immunisation at 2 and 4 months of age, 23 of the $32(71.9 \%)$ children presenting with AEFIs were admitted to hospital for AEFIs in the post-4CMenB era (13 to the ward and 10 to the CDU); thus meeting a WHO criteria for a serious AEFI. This compares with 7 of 17 $(41.2 \%)$ in the pre-4CMenB era ( 4 to the ward, 3 to the $\mathrm{CDU}$ ), representing an increase in rates of ward admissions from 0.14 (in the pre-4CMenB era) to 0.96 (in the post-4CMenB era) per 1000 immunisation episodes, and to the CDU from $0.10 / 1000$ to $0.74 / 1000$ (Figure 2a)). 
Lumbar punctures were performed on $21.9 \%$ of children 2 and 4 months of age presenting with AEFIs in the post-4CMenB era (7/32), compared with $17.6 \%$ in the pre-4CMenB era (3/17), an increase from 0.1 to 0.52 per 1000 immunisation episodes (Figure 2a)). In the post4CMenB era, the likelihood of receiving a lumbar puncture was similar at 2 and 4 months of age (5/25 (20\%) and 2/7(28.6\%) respectively).

Venepuncture (for blood cultures, full blood counts and/or C-reactive protein) was performed in 12/32 (37.5\%) of children presenting with AEFIs following 4CMenB immunisation at 2 and 4 months, compared to $6 / 17$ (35.3\%) in the pre-4CMenB era, an increase from 0.21 to 0.89 per 1000 immunisation episodes (Figure 2a)). Rates of IV antibiotics were similarly increased (Figure 2a)).

By contrast, Figure $2 \mathrm{~b}$ ) shows that there was no statistically significant increase in rates of admissions, lumbar punctures, blood tests or intravenous antibiotic use after immunisation at 3 months of age in the pre- versus post-4CMenB era (not including children presenting with 4CMenB administered at 3 months in the 'catch-up' campaign).

\section{Discussion}

This study has shown an increase in presentations to A\&E for AEFIs since the introduction of the 4CMenB vaccine from 1 in 972 doses to 1 in 295 at the 2-month immunisation episode, and from 1 in 7043 doses to 1 in 881 at the 4-month immunisation episode. This has been associated with an increase in hospital admissions (classified as serious AEFIs), invasive investigations and use of intravenous antibiotics. 
Despite this being a retrospective study, the risk of overlooking any presentations due to AEFIs was minimised by reviewing all relevant presentations in the age groups of interest, rather than relying on clinician assessment alone. Conversely, any child with an identified alternative cause for their symptoms (e.g. microbiologically confirmed sepsis or meningitis) was excluded. Nevertheless an element of diagnostic uncertainty remains, particularly as some infants were treated for sepsis. However, a real and causally related increase in AEFI presentations following 4CMenB introduction is supported by the consistency of assessment methods used for the pre- and post-4CMenB eras, the lack of changes in presentations due to a control condition (UTIs) and the specificity of the increases to the 2 and 4 month immunisation episodes, as opposed to 3 months (when no 4CMenB immunisation is given). Also, while calculations about rates of AEFI's per vaccines administered requires assumptions that all children immunised in Oxfordshire sought emergency department management in this county, and that no children seeking emergency care in Oxfordshire had been immunised elsewhere, it is expected that these factors would be unchanged in the pre- and post-4CMenB eras.

While these data reflect the first 'real life' evidence on the impact on hospital health care consumption of AEFIs observed following routine introduction of the 4CMenB vaccine, relevant data were obtained in a phase 3 study evaluating all medically attended postimmunisation fever in a 2, 4 and 6 month schedule of $4 \mathrm{CMenB}$ and routine vaccines. (7) Overall rates of medically attended fever following immunisation were higher in this trial than in our study (21.9/1000 following immunisation episodes containing 4CMenB) likely reflecting its inclusion of primary care as well as hospital services. Strikingly, despite lower rates of fever in children immunised with routine vaccines than after $4 \mathrm{CMenB}$ and routine vaccines, rates of medically attended fever in the routine-only group were at least as high as those after 4CMenB (22.14/1000 immunisation episodes). An increase in medically attended fever following 
4CMenB was observed only in a 'blinded' sub-group within this study by Vesikari et al, raising the possibility that parental education about the likelihood of post-immunisation fever might reduce subsequent health seeking behaviour. It was also anticipated that medically attended AEFIs could be minimised by recommendations to use prophylactic paracetamol at the time of immunisation as this was shown to reduce rates of fever $\left(\geq 38.5{ }^{\circ} \mathrm{C}\right)$ from $70.3 \%$ to $39.1 \%$ without impacting on vaccine immunogenicity $(4,8)$. Whether or not parents presenting their child to A and E with AEFIs had followed this advice was not determined in this study due to its retrospective nature but is an area that warrants further research. Also relevant to the use of paracetamol around the time of immunisation are two studies suggesting that this could impact on vaccine immunogenicity (8), (9). Of note is that neither of these studies involved $4 \mathrm{CMenB}$, and the one study evaluating the impact of paracetamol on the immunogenicity of a schedule that included 4CMenB showed no significant effect (10).

While additional research is required to confirm these findings across a great number of hospitals, and over a longer period of time, our data suggest that clinicians will have to manage 2 month olds presenting with AEFIs more frequently. A particular challenge for clinicians is distinguishing AEFIs from co-incidental sepsis in this vulnerable age group (for which full septic screens are recommended for fever without a focus). (3) We have not attempted to differentiate these presentations in this study (and any children with confirmed sepsis were excluded from our analysis) however further research to guide decision making and guideline development in this area may reduce the number of invasive investigations performed in these infants. As the vaccine only started being given to 12-month-old children in May 2016, it would also be of interest to study both the rates of AEFIs and the impact of the known risk of febrile seizures at this age. (7) Preliminary results from a general practise database survey led by 
Public Health England also suggest an increase in primary care consultations and this research is ongoing. (11)

These data should not detract from the success of the 4CMenB immunisation campaign, which has halved the number of cases of group B meningococcal disease in the immunised cohort (1). It should also be emphasised that the AEFIs described here are temporary and would not be expected to have long-term consequences, in contrast to the devastating impact that invasive meningococcal disease can have. Nevertheless, countries considering routine introduction of 4CMenB should expect an increase in AEFI related medical consultations, and further research is required to determine how these can be minimised.

\section{Acknowledgments}

The authors wish to thank Karyn Gokcil and Annette Sandrawich from the Oxfordshire Child Health Information Services - South, Central and West CSU for their assistance in determining immunisation histories.

\section{Ethics}

Permission to conduct this study as a 'service evaluation' was obtained through the OUH NHS trust, thus the study did not require approval from an NHS ethics committee. Low risk ethical approval was granted by Monash University.

\section{Funding}

This project was internally funded. We have received support from the NIHR Oxford Biomedical Research Centre and a travel grant from Monash University Department of Paediatrics. 


\section{Copyright}

I, Viveka Nainani, affirm that the manuscript is an honest, accurate, and transparent account of the study being reported; that no important aspects of the study have been omitted; and that any discrepancies from the study as planned have been explained.

\section{What is already known on this topic?}

The $4 \mathrm{CMenB}$ vaccine has been known to have post immunisation fever rates of approximately 61\%. There have been no studies assessing the numbers of infants presenting to A\&E with fever due to the $4 \mathrm{CMenB}$ vaccine as compared to routine vaccinations.

\section{What this study adds}

This project has shown an increase in the number of children presenting to accident and emergency, admitted to hospital, receiving investigations and IV antibiotics due to AEFIs post4CMenB introduction. This uncertainty in management highlights the need to develop guidelines for these infants when they present to A\&E to minimise increased healthcare consumption. 


\section{References}

1. McKee S. Real world data back efficacy of GSK's Bexsero. 2016 [updated 6 September 2016. Available from:

http://www.pharmatimes.com/news/real world data back efficacy of gsks bexsero 1 $\underline{122744 .}$.

2. Gossger N, Snape MD, Yu LM, Finn A, Bona G, Esposito S, et al. Immunogenicity and tolerability of recombinant serogroup B meningococcal vaccine administered with or without routine infant vaccinations according to different immunization schedules: a randomized controlled trial. JAMA. 2012;307(6):573-82.

3. National Institute for Health and Care Excellence. Fever in under 5s: assessment and initial management May 2013 [Available from:

https://www.nice.org.uk/guidance/cg160/chapter/1-recommendations.

4. Public Health England. Using paracetamol to prevent and treat fever after MenB vaccination: National Health Service; [Available from:

https://www.gov.uk/government/uploads/system/uploads/attachment data/file/483 408/9413-paracetamol-menB-2page-A4-08-web.pdf.

5. Public Health England. The UK immunisation schedule 2015 [updated February 2015. Available from:

https://www.gov.uk/government/uploads/system/uploads/attachment data/file/400 554/2902222 Green Book Chapter 11 v2 4.pdf.

6. World Health Organisation. Global manual on surveillance of adverse events following immunization. 2014.

7. Vesikari T, Esposito S, Prymula R, Ypma E, Kohl I, Toneatto D, et al.

Immunogenicity and safety of an investigational multicomponent, recombinant, meningococcal serogroup B vaccine (4CMenB) administered concomitantly with routine infant and child vaccinations: results of two randomised trials. Lancet. 2013;381(9869):825-35.

8. Prymula R, Siegrist CA, Chlibek R, Zemlickova H, Vackova M, Smetana J, et al. Effect of prophylactic paracetamol administration at time of vaccination on febrile reactions and antibody responses in children: two open-label, randomised controlled trials. Lancet. 2009;374(9698):1339-50.

9. Wysocki J, Center KJ, Brzostek J, Majda-Stanislawska E, Szymanski H, Szenborn L, et al. A randomized study of fever prophylaxis and the immunogenicity of routine pediatric vaccinations. Vaccine. 2017;35(15):1926-35.

10. Prymula R, Esposito S, Zuccotti GV, Xie F, Toneatto D, Kohl I, et al. A phase 2 randomized controlled trial of a multicomponent meningococcal serogroup $B$ vaccine (I). Hum Vaccin Immunother. 2014;10(7):1993-2004

11. Harcourt S, Morbey R, Bates C, Carter H, Ladhani S, Elliot A J, Smith GE. Infant fever trends following the launch of the meningococcal B vaccine in the UK. Online J Pub Health Infomatics. 2017; 9 (1). 


\section{Tables and Figures}

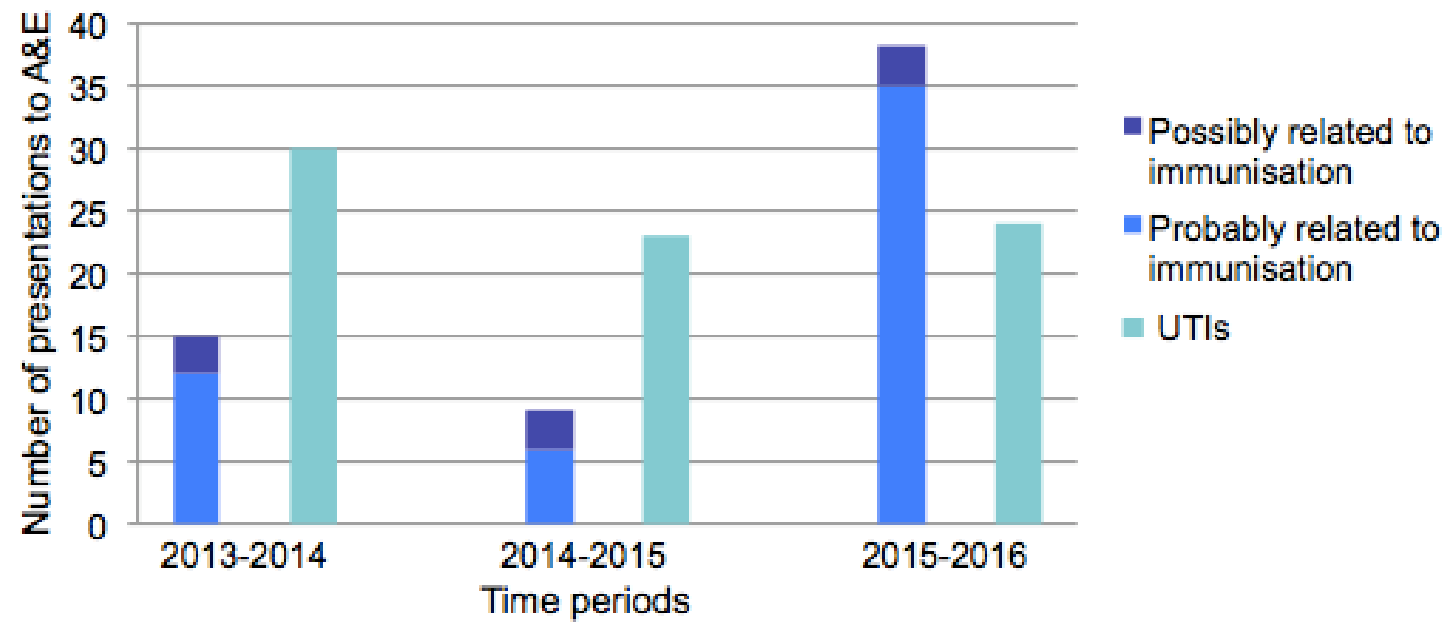

Figure 1: Number of presentations to A and E for probable and possible AEFIs, and for UTIs, per year

Table 1: AEFIs by age at immunisation, and expressed in terms of numbers of doses of routine or 4 CMenB vaccines

\begin{tabular}{|c|c|c|c|c|c|c|}
\hline Age & Era & $\begin{array}{c}\text { Doses } \\
\text { administered* }\end{array}$ & $\begin{array}{c}\text { Probable/ } \\
\text { possible } \\
\text { AEFIs }\end{array}$ & AEFIs per 1000 doses & OR (95\% CI) & p-value \\
\hline \multirow[t]{2}{*}{2 months } & Pre-4CMenB & 14584 & 15 & 1.03 & $3.3(1.7,6.3)$ & $<0.001$ \\
\hline & Post-4CMenB & 7381 & 25 & 3.39 & & \\
\hline \multirow[t]{2}{*}{3 months } & Pre-4CMenB & 14741 & 7 & 0.47 & $1.7(0.6,5.1)$ & 0.380 \\
\hline & Post-4CMenB & 7381 & 6 & 0.81 & & \\
\hline $\begin{array}{l}3 \text { months } \\
\text { (catch up) }\end{array}$ & NA & 592 & 4 & 6.76 & - & - \\
\hline \multirow[t]{2}{*}{4 months } & Pre-4CMenB & 14086 & 2 & 0.14 & $8.0(1.7,38.5)$ & 0.005 \\
\hline & Post-4CMenB & 6168 & 7 & 1.13 & & \\
\hline \multirow{2}{*}{$\begin{array}{l}2 \& 4 \text { Months } \\
\text { combined }\end{array}$} & Pre-4CMenB & 28670 & 17 & 0.59 & $4.0(2.2,7.2)$ & $<0.001$ \\
\hline & Post-4CMenB & 13549 & 32 & 2.36 & & \\
\hline
\end{tabular}

* Doses refers to combination diphtheria-toxoid, tetanus toxoid, acellular pertussis, inactivated polio and Haemophilus influenzae type b at 2, 3 and 4 months in the pre-4CMenB era, and 3 months on the post-4CMenB era, and 4CMenB at 2 and 4 months and 3 months (catch up) in the post-4CMenB era. 
Rates (per 1000) and Odds Ratios (95\% Cl) for AEFIs

a)

2 \& 4 month immunisation episodes combined

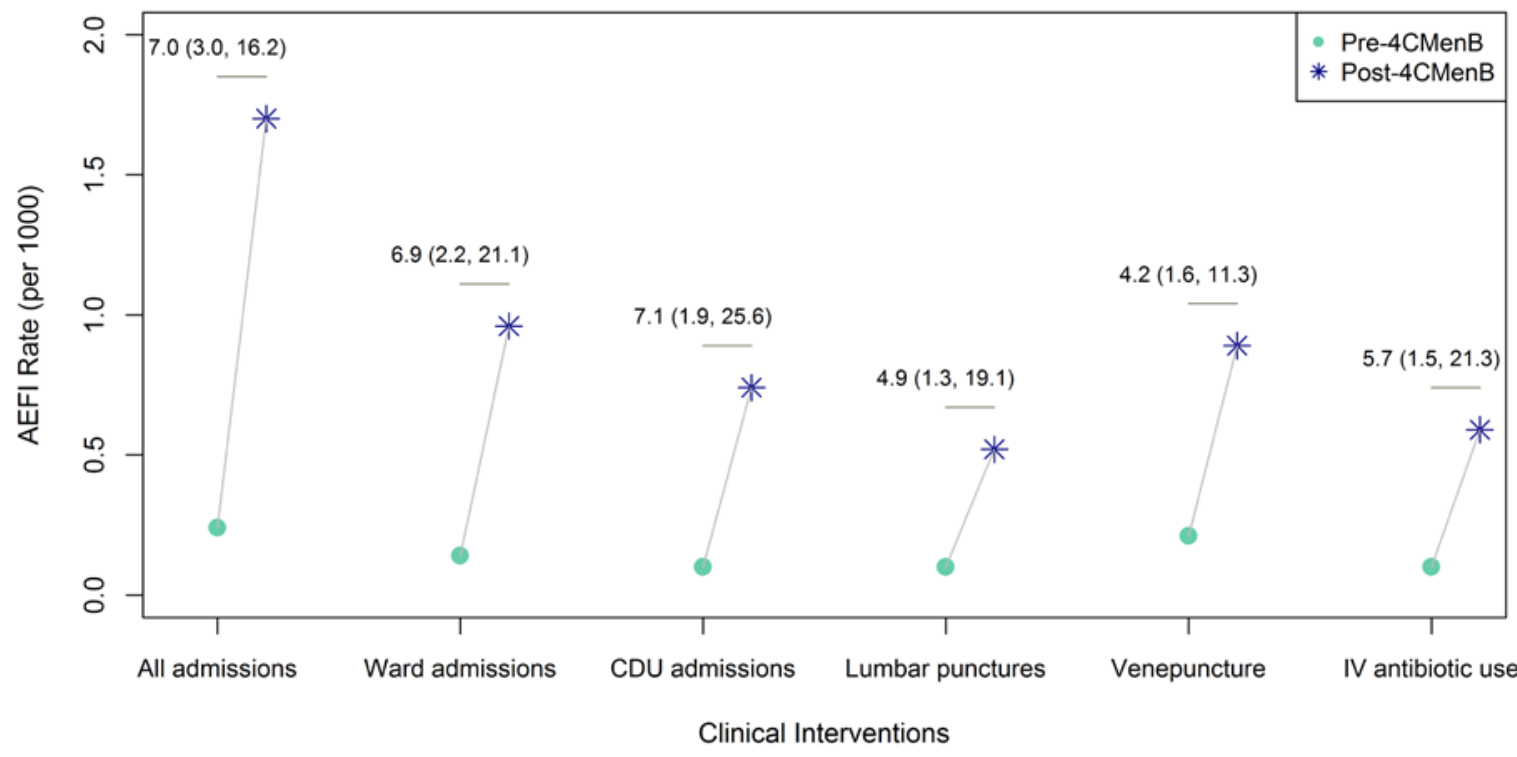

b)

3 month immunisation episodes

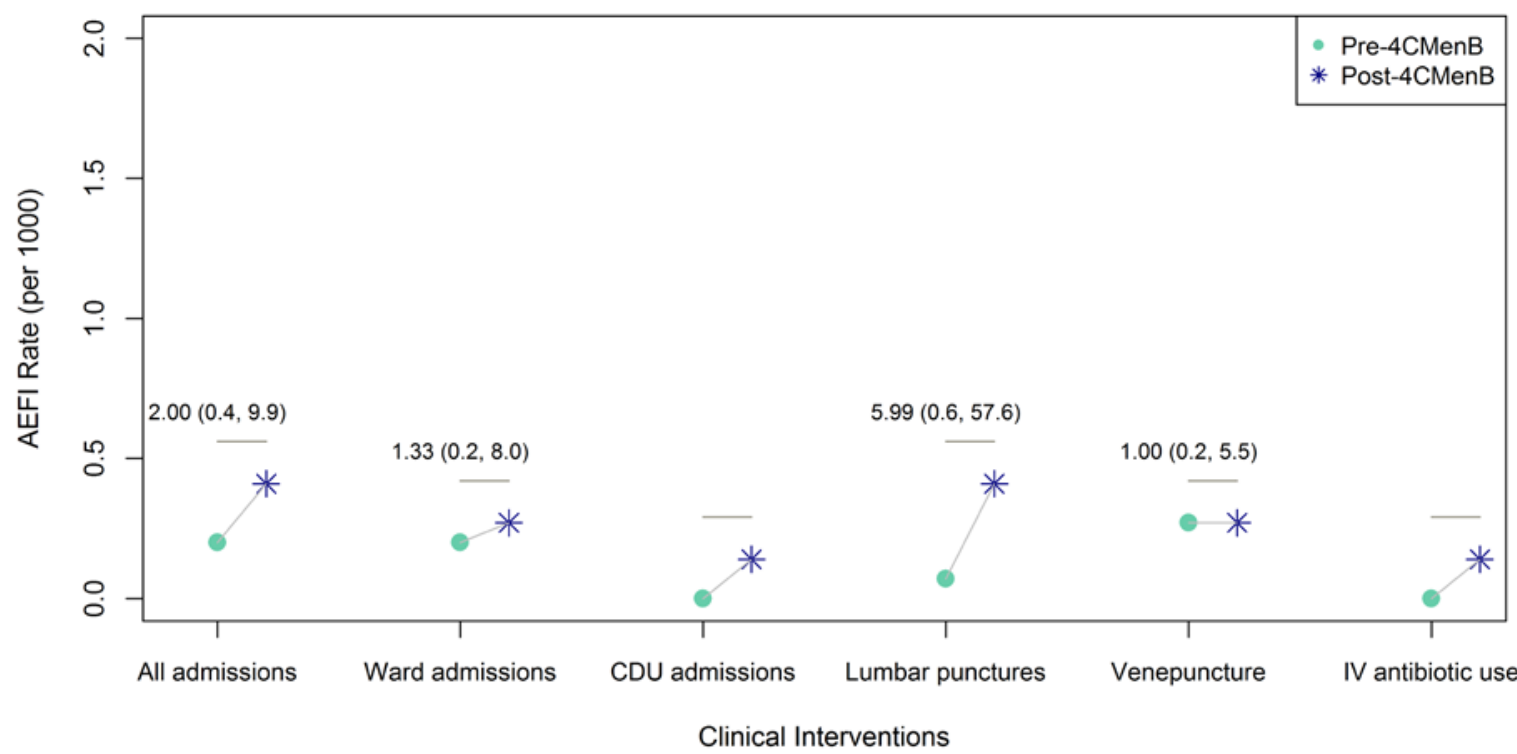

Figure 2: Rates of clinical intervention for probable or possible AEFIs per 1000 doses of vaccine by pre-/post-4CMenB era, for: a) 2 and 4 month immunisation episodes combine and b) 3 month immunisation episodes. Odds Ratios (ORs) and 95\% confidence intervals are presented above the $\mathrm{x}$-axis in each plot. There are no ORs for when there were no cases. 


\section{Supplementary Table 1:}

\begin{tabular}{|c|c|c|}
\hline Age due & Pre-4CMenB era & Post-4CMenB era \\
\hline $\begin{array}{l}\text { Two } \\
\text { months } \\
\text { old }\end{array}$ & $\begin{array}{l}\text { - Diphtheria, tetanus, pertussis, polio and } \\
\text { Haemophilus influenza type b Hib } \\
\text { (DTaP/IPV/Hib) } \\
\text { - Pneumococcal conjugate (PCV) } \\
\text { - Rotavirus }\end{array}$ & $\begin{array}{l}\text { - Diphtheria, tetanus, pertussis, polio and } \\
\text { Haemophilus influenza type b Hib } \\
\text { (DTaP/IPV/Hib) } \\
\text { - Pneumococcal conjugate (PCV) } \\
\text { - Rotavirus } \\
\text { - Meningococcal group B (4CMenB) }\end{array}$ \\
\hline $\begin{array}{l}\text { Three } \\
\text { months } \\
\text { old }\end{array}$ & $\begin{array}{l}\text { - Diphtheria, tetanus, pertussis, polio and } \\
\text { Hib (DTaP/IPV/Hib) } \\
\text { - Meningococcal C conjugate (MenC) } \\
\text { - Rotavirus }\end{array}$ & $\begin{array}{l}\text { - Diphtheria, tetanus, pertussis, polio and } \\
\text { Hib (DTaP/IPV/Hib) } \\
\text { - Meningococcal C conjugate (MenC)* } \\
\text { - Rotavirus }\end{array}$ \\
\hline $\begin{array}{l}\text { Four } \\
\text { months } \\
\text { old }\end{array}$ & $\begin{array}{l}\text { - Diphtheria, tetanus, pertussis, polio and } \\
\text { Hib (DTaP/IPV/Hib) } \\
\text { - Pneumococcal conjugate (PCV) }\end{array}$ & $\begin{array}{l}\text { - Diphtheria, tetanus, pertussis, polio and } \\
\text { Hib (DTaP/IPV/Hib) } \\
\text { - Pneumococcal conjugate (PCV) } \\
\text { - Meningococcal group B (4CMenB) }\end{array}$ \\
\hline
\end{tabular}

Supplementary Table 1: Routine immunisation schedule in the UK up to 4 months of age, pre and post-introduction of the 4 CMenB vaccine. (5)

* MenC vaccine was removed from 3 month UK immunisation schedule in July 2016 


\section{Supplementary Figure 1-2:}

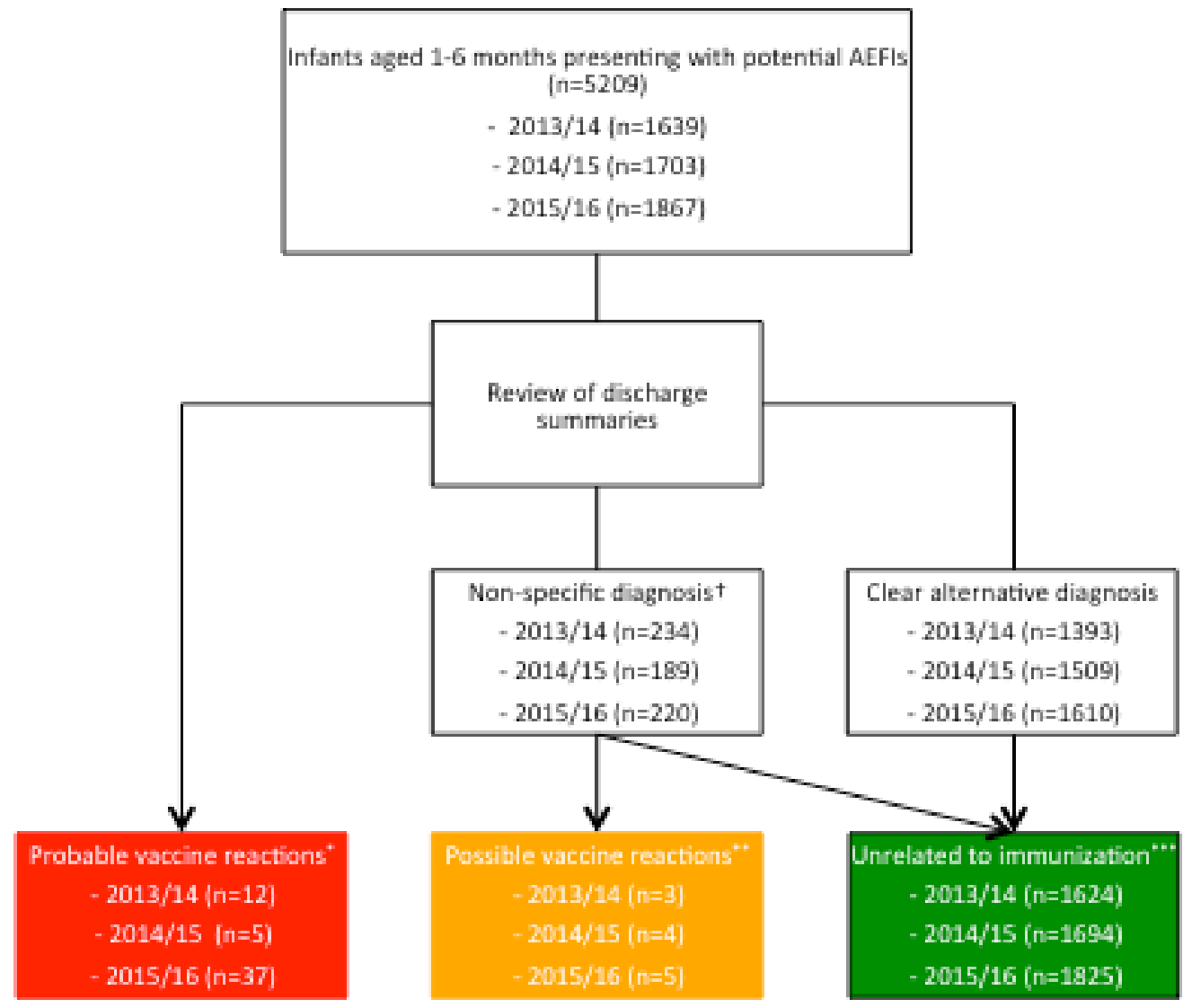

'Crying, irritability, sepsis, flappiness, unwel, fever or 'conditions of interest' [meningitis, seizure, rash]

"Discharge summaries and/or clinical notes report vaccine reaction as clinical diagnosis

" Records show no clear alternative diagnasis obtained and immunisation occurred within previaus 48 hours

$\cdots$ Clear alternative diagnosis/conditions that are unlikely to be related to immunisation or no immunisation within previous 48 hours

Supplementary Figure 1: Classification of infants aged 1-6 months presenting to Accident and Emergency in 2013-14, 2014-15 and 2015-16 


\section{Annual rate of presentations to A\&E per 1000 doses}

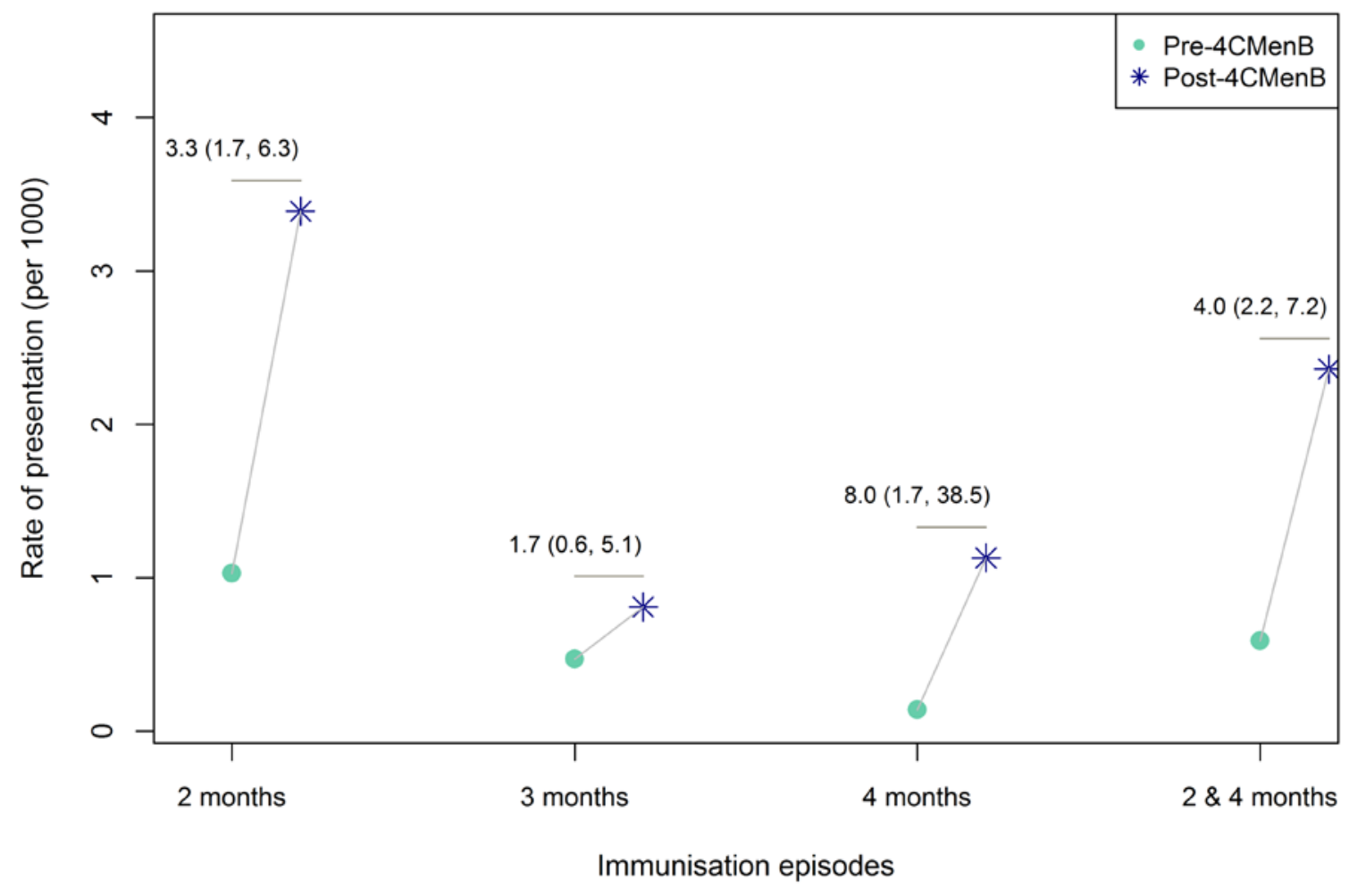

Supplementary Figure 2: Annual rate of presentations to A\&E for probable or possible AEFIs per 1000 doses, for different age groups, by pre/post-4CMenB era 